\title{
Uncertainty quantification for a model of HIV-1 patient response to antiretroviral therapy interruptions
}

\author{
Robert Baraldi $^{1}$, Karissa Cross ${ }^{1}$, Christina McChesney ${ }^{1}$, Laura Poag ${ }^{1}$, Emma Thorpe ${ }^{1}$ \\ Kevin B. Flores ${ }^{1}$ and H.T. Banks ${ }^{1, *}$
}

\begin{abstract}
We consider a model for in-host HIV-1 infection dynamics developed and validated with patient data in earlier work[1]. We revisit the earlier model in light of progress over the last several years in understanding of HIV-1 progression in humans. We then consider statistical models to describe the data and use these with residual plots in weighted least squares problems to develop accurate descriptions of the proper weights for the data. Bootstrapping is then used to develop confidence intervals for the resulting parameter estimates and establish absence of correlation in the estimated parameters.
\end{abstract}

\section{INTRODUCTION}

Individuals infected with Human Immunodeficiency Virus (HIV-1) and subsequently treated with antiretroviral therapy (ART) can have disparate outcomes. A recent study showed that 11 HIV-1 patients maintained low HIV-1 levels without ART after being treated with ART for an average of 36.5 months [2]. However, in the majority of cases, HIV-1 patients must be treated with ART indefinitely, living with toxic side effects [3], [4] and with the potential risk of developing Acquired Immunodeficiency Syndrome (AIDS) [5]. Several factors have been elucidated that can help explain how HIV1 escapes the action of ART and leads to disparities in ART outcomes.

One major factor that has been of particular interest recently is the ability of HIV-1 to lay dormant in so called "reservoir cells". In this scenario, a subset of infected cells harbor the virus in a latent state in which the virus is not produced at all or at undetected levels [6], [7]. Since ART targets the various stages of viral replication, HIV-1 that is integrated into the DNA of reservoir cells can evade ART and reseed the HIV-1 infection when patients are taken off ART [8], [1], [9].

There is evidence that the presence and size of cellular HIV-1 reservoirs are associated with the ability of the patient to control the HIV-1 infection, as well as external factors such as the timing and duration of ART. For example, some patients called "elite controllers" possess the innate ability to suppress the HIV-1 infection without ART. In these patients, the HIV-1 reservoir is significantly lower than in patients that require ART [10], [11], [12]. Among HIV-1 patients

This research was supported in part by grant number NIAID R01AI071915-10 from the National Institute of Allergy and Infectious Diseases, in part by the National Science Foundation by Undergraduate Biomathematics grant number DBI-1129214 and in part by the National Science Foundation under grant number DMS-0946431.

${ }^{1}$ Center for Research in Scientific Computation, Center for Quantitative Sciences in Biomedicine, North Carolina State University, Raleigh, NC, USA

*Corresponding author, htbanks@ncsu.edu that require ART, those treated during the earliest stages of infection have a smaller reservoir than those treated at later stages [13]. Furthermore, some patients that are able to suppress HIV-1 after the termination of long-term ART therapy have low HIV-1 reservoirs in central-memory CD4 $\mathrm{T}$ cells [2]. These clinical findings emphasize the need to quantitate the interaction between HIV-1 replication, the host immune system, latent reservoirs, and disease stage in a patient-specific manner. Such a quantitation could enable more accurate predictions of how patients will respond to ART and lead to personalized treatment regimens.

In 2007, Banks et al published a mathematical model that was used to quantitate the interaction between HIV-1 and the immune system on an individual patient level [1]. They performed this quantitation by estimating parameters for their mathematical model using longitudinal data from a clinical study involving structured treatment interruptions (STIs) in which patients were cycled on and off ART for some duration of time. Their model captured the transient viremia experienced by some patients on therapy with viral load levels suppressed below the detection limit, i.e. the reemergence of virus from latently infected cells once treatment successfully reduced the level of free virus.

While Banks et al found that some of their model parameters could be taken as constant across patients, other parameters, including some describing the latent HIV-1 reservoir, varied between patients. Since these parameters were used to predict different HIV-1 patient responses to STIs, it is important to quantify the confidence in their estimation. The assessment of parameter uncertainty is a highly nonlinear problem that depends on the mathematical model, the statistical error model for parameter estimation, and the data set. Although parameter uncertainty quantification is widely held as an important step in the modeling process, and despite the fact that there are multiple methods for computing uncertainty [14], it may not be performed in the first stages of the modeling process. These first stages are often focused on the predictive capability and biological accuracy of the model. Since parameter uncertainty was not assessed for the Banks et al HIV-1 model, we perform this assessment here and discuss the resulting biological implications of our findings.

We examine the uncertainty in parameter estimates for data from a single patient that underwent three ART interruptions. First, because the field of HIV-1 biology has developed rapidly since the Banks et al model was published, we provide an in depth model description to justify that 
the Banks et al HIV-1 model is current. We proceed by describing the data and inverse problem methodology. We then analyze the statistical error model and use these results to perform parameter estimation and quantify parameter uncertainty with bootstrapping. We also test for correlations between parameters estimates.

\section{MODEL DESCRIPTION AND VERIFICATION}

A system of ordinary differential equations has been outlined by Banks et al [1] to describe the pathogenesis of HIV-1 infection (Eq. (1) - (8)), including the the role of CD4 memory cells that serve as a major reservoir of latently infected cells. This model does not reflect the nature of all immune responses and all host and viral dynamics but instead captures the most prominent biological features. It has also been shown to accurately recapitulate the within host dynamics of CD4 T cells and virus levels from clinical patient data [1].

The biological model is given in Figure 1 , where $T_{1}$ represents uninfected activated (antigen specific) CD4 T cells and $T_{1}^{*}$ is infected activated CD4 T cells. Uninfected resting, or not activated, CD4 T cells are represented by $T_{2}$ and infected resting CD4 $\mathrm{T}$ cells are given by $T_{2}^{*}$. Infectiousfree virus (virus that is capable of infecting other cells) is represented by $V_{I}$ and non-infectious-free virus (virus that is yielded inactive by protease inhibitors) is represented by $V_{N I}$. HIV-1-specific effector CD8 $\mathrm{T}$ cells are given by $E_{1}$ and HIV-1-specific memory CD8 T cells are represented by $E_{2}$.

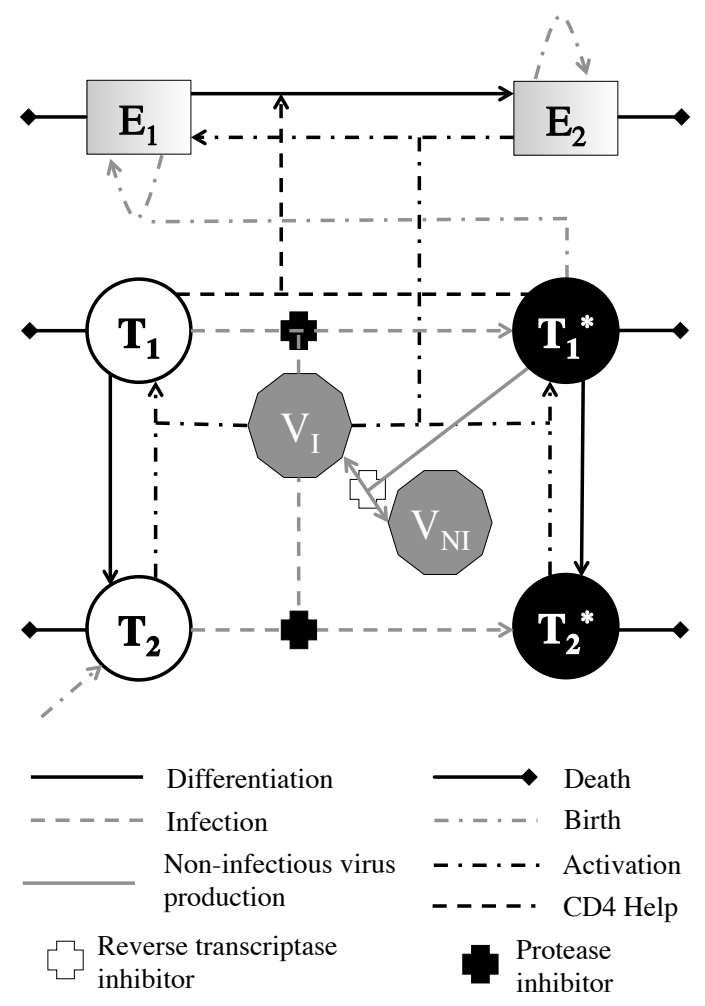

Fig. 1. Compartmental diagram for the mathematical model given in [1].

$$
\begin{aligned}
\dot{T}_{1} & =-d_{1} T_{1}-\left(1-\xi_{1}(t)\right) k_{1} V_{I} T_{1}-\gamma_{T} T_{1} \\
& +p_{T}\left(\frac{a_{T} V_{I}}{V_{I}+K_{V}}+a_{A}\right) T_{2} \\
\dot{T}_{1}^{*}= & \left(1-\xi_{1}(t)\right) k_{1} V_{I} T_{1}-\delta T_{1}^{*}-m E_{1} T_{1}^{*}-\gamma_{T} T_{1}^{*} \\
& +p_{T}\left(\frac{a_{T} V_{I}}{V_{I}+K_{V}}+a_{A}\right) T_{2}^{*} \\
\dot{T}_{2}= & \lambda_{T} \frac{K_{s}}{V_{I}+K_{s}}+\gamma_{T} T_{1}-d_{2} T_{2} \\
- & \left(1-f \xi_{1}(t)\right) k_{2} V_{I} T_{2}-\left(\frac{a_{T} V_{I}}{V_{I}+K_{V}}+a_{A}\right) T_{2} \\
\dot{T}_{2}^{*}= & \gamma_{T} T_{1}^{*}+\left(1-f \xi_{1}(t)\right) k_{2} V_{I} T_{2}-d_{2} T_{2}^{*} \\
- & \left(\frac{a_{T} V_{I}}{V_{I}+K_{V}}+a_{A}\right) T_{2}^{*} \\
\dot{V}_{I}= & \left(1-\xi_{2}(t)\right) 10^{3} N_{T} \delta T_{1}^{*}-c V_{I} \\
- & 10^{3}\left[\left(1-\xi_{1}(t)\right) \rho_{1} k_{1} T_{1}+\left(1-f \xi_{1}(t)\right) \rho_{2} k_{2} T_{2}\right] V_{I} \\
\dot{V}_{N I}= & \xi_{2}(t) 10^{3} N_{T} \delta T_{1}^{*}-c V_{N I} \\
\dot{E}_{1}= & \lambda_{E}+\frac{b_{E 1} T_{1}^{*}}{T_{1}^{*}+K_{b 1}} E_{1}-\frac{d_{E} T_{1}^{*}}{T_{1}^{*}+K_{d}} E_{1}-\delta_{E 1} E_{1} \\
- & \gamma_{E} \frac{T_{1}+T_{1}^{*}}{T_{1}+T_{1}^{*}+K_{\gamma}} E_{1}+\frac{p_{E} a_{E} V_{I}}{V_{I}+K_{V}} E_{2} \\
& \quad \delta_{E 2} E_{2}-\frac{a_{E} V_{I}}{V_{I}+K_{V}} E_{2} \\
& \dot{E}_{2}=\gamma_{E} \frac{T_{1}+T_{1}^{*}}{T_{1}+T_{1}^{*}+K_{\gamma}} E_{1}+\frac{b_{E 2} K_{b 2}}{E_{2}+K_{b 2}} E_{2} \\
& \quad a
\end{aligned}
$$

with initial condition vector $\left[T_{1}(0), T_{1}^{*}(0), T_{2}(0), T_{2}^{*}(0), V_{I}(0), V_{N I}(0), E_{1}(0), E_{2}(0)\right]$. For an in-depth explanation of the model and parameter definitions, refer to [1].

We attempted to use current knowledge to support the accuracy of this model. We investigated six assumptions: that (i) the proliferation of CD4 and the activation of CD8 memory $\mathrm{T}$ cells is correctly represented, (ii) the role of CD4 cell-produced interferons play in CD8 cell differentiation is properly included, (iii) the interaction between infected CD4 T cells and immune effector cells is accurate, and (iv) the mechanism by which activation of resting CD4 T cells occurs is consistent with recent research.

Assumption (i) is supported by a 2011 paper by Catalfamo et al which describe that naive CD4 $\mathrm{T}$ cells are mainly recruited in response to $\mathrm{CD} 4 \mathrm{~T}$ cell depletion whereas CD8 memory $\mathrm{T}$ cell activation is driven mainly by HIV-1 RNA levels [15]. These mechanisms are modeled by the density dependent regulation of CD4 cells by the term $\lambda_{T} \frac{K_{s}}{V_{I}+K_{s}}-d_{2} T_{2}$ in equation (3) which increases as CD4 levels decrease. Viral load level is also incorporated into this term to reflect the diminished thymic production of CD4 cells when viral load is too high [1]. The HIV-1 RNA dependent activation of CD8 memory cells into CD8 effector cells is incorporated into the model by the term $\frac{p_{E} a_{E} V_{I}}{V_{I}+K_{V}} E_{2}$, where virus level promotes the activation of 
CD8 memory cells $\left(E_{2}\right)$, adding to the pool of CD8 effector cells $\left(E_{1}\right)$. Assumption (ii) is supported by three recent papers which describe that Type 1 interferons are cytokines that are induced directly by virus infection, which in turn promote the proliferation of antigen-specific CD8 $\mathrm{T}$ cells [15], [16], [17]. This role is represented by the dotted line from $T_{1}$ and $T_{1}^{*}$ to the differentiation of immune effector cells $\left(E_{1}\right)$ into immune memory cells $\left(E_{2}\right)$ in figure 1 and the corresponding term $\frac{T_{1}+T_{1}^{*}}{T_{1}+T_{1}^{*}+K_{\gamma}} E_{1}$ in equations (6) and (7). Assumption (iii) is supported by a 2011 paper which showed that the presence of $T_{1}^{*}$ stimulates the proliferation of $E_{1}$ through antibody presentation [18] and contributes to the the $E_{1}$ death rate due to the mechanism of activationinduced cell death[19]. Therefore the net effect of these terms on $\dot{E}_{1}$ is a function of $T_{1}^{*}$. For $T_{1}^{*}<\frac{K_{b 1} d_{E}-b_{E 1} K_{d}}{b_{E 1}-d_{E}}$, the terms $\frac{b_{E 1} T_{1}^{*}}{T_{1}^{*}+K_{b 1}}$ and $\frac{-d_{E} T_{1}^{*}}{T_{1}^{*}+K_{d}}$ contribute positively to $\left(\dot{E}_{1}\right)$, representing early infection when antigen presentation by emerging $T_{1}^{*}$ stimulates the production of effector cells. For $T_{1}^{*}>\frac{K_{b 1} d_{E}-b_{E 1} K_{d}}{b_{E 1}-d_{E}}$, these terms contribute negatively to $\dot{E}_{1}$. Assumption (iv) is supported by two recent papers that describe dendritic cell (DC) mediated activation of HIV-1 latent reservoir cells [20], [21]. In this scenario, the amount of virus can directly affect the activation of HIV-1-specific resting CD4 $\mathrm{T}$ Cells by affecting the amount of antigen presenting cells, including DCs.

\section{DATA AND METHODS}

\section{A. Data sets}

Data used by Banks et al includes patients from Massachusetts General Hospital that underwent combination therapy, many of which had at least one treatment interruption[1]. The collected data for each patient are total CD4 T cell count $/ \mu$ L-blood and total RNA copies $/ \mathrm{mL}$ plasma. If there are less than 400 copies/mL-plasma for a standard assay or 50 copies/mL-plasma for an ultra-sensitive assay, then the viral load value is censored to be the limit of quantification. For these data points, the expectation maximization (EM) algorithm was applied in [1]. For this work, we chose to model the data for Patient 1 from Banks et al because the mathematical model was very accurate for over four years. The EM data points for Patient 1 were combined with the uncensored data to form a data set that could be used for parameter estimation. In total, there were 102 data points for viral load and 84 data points for the CD4 $\mathrm{T}$ cell count over 1527 days.

\section{B. Inverse problem methodology}

In order to estimate a vector of parameters $q$, we use the data for CD4 $\mathrm{T}$ cell count, $\left\{y_{1}^{i}\right\}_{i=1}^{N_{1}}$, and data for the viral load, $\left\{y_{2}^{i}\right\}_{i=1}^{N_{2}}$. The mathematical model divides total CD4 T cells into uninfected activated $\left(T_{1}\right)$, infected activated $\left(T_{1}^{*}\right)$, uninfected resting $\left(T_{2}\right)$, and infected resting $\left(T_{2}^{*}\right)$, so the total CD4 T cell count is represented by $z_{1}=T_{1}+T_{1}^{*}+T_{2}+T_{2}^{*}$. Similarly, since RNA copies are separated into infectiousfree virus $\left(V_{I}\right)$ and non-infectious-free virus $\left(V_{N I}\right)$, total RNA copies is represented by $z_{2}=V_{I}+V_{N I}$. We used a least squares framework with the following statistical error models for the observables $z_{1}$ and $z_{2}$ :

$$
\begin{array}{r}
Y_{1}^{i}=z_{1}\left(t_{1}^{i}, \vec{q}\right)+e_{1}^{i}, i=1,2, \ldots, N_{1}, \\
Y_{2}^{i}=z_{2}\left(t_{2}^{i}, \vec{q}\right)+z_{2}^{\gamma}\left(t_{2}^{i}, \vec{q}\right) e_{2}^{i}, i=1,2, \ldots, N_{2},
\end{array}
$$

where $y_{j}^{i}$ are realizations of the random variable $Y_{j}^{i}$ and $e_{j}^{i}$ are i.i.d., and $\mathbb{E}\left[e_{j}^{i}\right]=0, \operatorname{Var}\left(e_{j}^{i}\right)=\sigma_{j}^{2}$ for $j=1,2$.

The least squares estimator is

$$
\begin{array}{r}
q_{0} \approx \hat{q}=\underset{q \in Q}{\arg \min }\left(\frac{1}{N_{1}} \sum_{i=1}^{N_{1}} \frac{\left(y_{1}^{i}-z_{1}\left(t_{1}^{i}, q\right)\right)^{2}}{\sigma_{1}^{2}}\right. \\
\left.+\frac{1}{N_{2}} \sum_{i=1}^{N_{2}} \frac{\left(y_{2}^{i}-z_{2}\left(t_{2}^{i}, q\right)\right)^{2}}{\sigma_{2}^{2} z_{2}^{2 \gamma}\left(t_{2}^{i}, q\right)}\right) \\
\sigma_{1}^{2}=\frac{1}{N_{1}+N_{2}-\operatorname{dim}(q)} \sum_{i=1}^{N_{1}}\left(y_{1}^{i}-z_{1}\left(t_{1}^{i}, q\right)\right)^{2} \\
\sigma_{2}^{2}=\frac{1}{N_{1}+N_{2}-\operatorname{dim}(q)} \sum_{i=1}^{N_{2}} \frac{\left(y_{2}^{i}-z_{2}\left(t_{2}^{i}, q\right)\right)^{2}}{z_{2}^{2 \gamma}\left(t_{2}^{i}, q\right)}
\end{array}
$$

Below, we discuss the need to weight the error for the viral load in proportion to the model solution and the value for the exponent $\gamma$ is specified. All inverse problems were performed in Matlab using the fminsearch function.

\section{Uncertainty quantification: Bootstrapping}

To implement bootstrapping to quantify the uncertainty in parameter estimates for equations (1) - (8) we first obtained a vector of estimated parameters, $\hat{q}$, using the above inverse problem methodology. We then calculated the standardized residuals, $\bar{r}_{j}^{i}$ for these estimates for each observable $(j=$ 1,2):

$$
\begin{aligned}
& \bar{r}_{1}^{i}=\sqrt{\frac{N_{1}}{N_{1}-\operatorname{dim}(\hat{q})}}\left(y_{1}^{i}-z_{1}\left(t_{1}^{i}, \hat{q}\right)\right), i=1,2, \ldots, N_{1} \\
& \bar{r}_{2}^{i}=\sqrt{\frac{N_{2}}{N_{2}-\operatorname{dim}(\hat{q})}}\left(\frac{y_{2}^{i}-z_{2}\left(t_{2}^{i}, \hat{q}\right)}{z_{2}^{\gamma}\left(t_{2}^{i}, \hat{q}\right)}\right), i=1,2, \ldots, N_{2}
\end{aligned}
$$

Bootstrap sample points were then created by sampling residuals with replacement from the set $\left\{\bar{r}_{j}^{i}\right\}_{i=1}^{N_{j}}$ for the observable $z_{j}, j=1,2$ and adding them to the model solution. We repeated this process $M=1000$ times to create 1000 data sets, each with 84 and 102 data points for CD4 T cell count and viral load, respectively. We then conducted an inverse problem on each of these 1000 data sets and stored the parameter estimates $\hat{q}^{m}(\mathrm{~m}=1, \ldots, 1000)$ in a matrix, $Q_{B O O T}$. With these values, the mean, variance, and standard errors for the parameters can be calculated using the following formulas given in [22], [14]: 


$$
\begin{aligned}
\hat{q}_{\text {BOOT }} & =\frac{1}{M} \sum_{m=1}^{M} \hat{q}^{m} \\
\operatorname{Var}\left(\hat{q}_{B O O T}\right) & =\frac{1}{M-1} \sum_{m=1}^{M}\left(\hat{q}^{m}-\hat{q}_{\text {BOOT }}\right)\left(\hat{q}^{m}-\hat{q}_{\text {BOOT }}\right)^{T}, \\
S E_{k}\left(\hat{q}_{\text {BOOT }}\right) & =\sqrt{\operatorname{Var}\left(\hat{q}_{\text {BOOT }}\right)_{k k}} .
\end{aligned}
$$

For each parameter $\hat{q}_{k}(\mathrm{k}=1, \ldots, \operatorname{dim}(\mathrm{q}))$ in our original parameter estimate vector $\hat{q}$, the $100(1-\alpha) \%$ confidence interval for $\hat{q}_{k}$ can be computed based on the standard errors associated with the parameter estimators $q_{W L S}$ which have an approximate asymptotic distribution

$q_{W L S} \sim \mathcal{N}\left(\hat{q}_{B O O T}, \operatorname{Var}\left(\hat{q}_{B O O T}\right)\right):$

$$
\begin{array}{r}
\operatorname{Prob}\left\{q_{k}-t_{1-\alpha / 2} S E_{k}(\hat{q})<q_{0 k}<q_{k}+t_{1-\alpha / 2} S E_{k}(\hat{q})\right\} \\
=1-\alpha,
\end{array}
$$

where $\alpha$ is chosen to be small (e.g., $\alpha=0.05$ for $95 \%$ confidence intervals) and $t_{1-\alpha / 2}$ is determined by $\operatorname{Prob}(T \geq$ $\left.t_{1-\alpha / 2}\right)=\alpha / 2$, where $T \sim t^{v}$ for $v=n-\operatorname{dim}(q)$ degrees of freedom [22]. The corresponding $95 \%$ confidence intervals are then given by

$$
\left[\hat{q}_{k}-t_{1-\alpha / 2} S E_{k}(\hat{q}), \hat{q}_{k}+t_{1-\alpha / 2} S E_{k}(\hat{q})\right] .
$$

\section{RESUlTS}

\section{A. Data fitting}

We fit our model to the CD4 T cell count and viral load data for Patient 1 by estimating a subset of parameters. Banks et al found that only 16 out of 39 total parameters in the model had significant variation between patients.
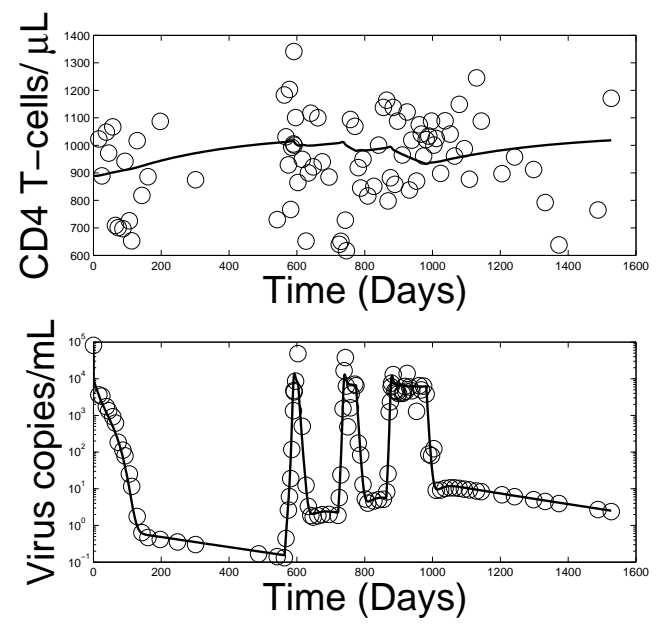

Fig. 2. The mathematical model (1) - (8) accurately tracks the dynamics of CD4 T cell and viral load for patient 1 over several years. Circles indicate data points and solid lines are the model simulations using parameter estimates found with setting $\gamma=1.2$.

We also fixed $V_{I}(0)$ to the initial viral load data point under the assumption that the amount of non-infectious virus is negligible at the time of treatment initiation. We argue that this assumption is reasonable because the primary cause of non-infectious production is ART (see the term $\xi_{2}(t)$ in Eq. (6)). Thus, we estimated the subset of 15 parameters $\left\{\lambda_{T}\right.$, $d_{1}, \varepsilon_{1}, k_{1}, a_{T}, \varepsilon_{2}, N_{T}, b_{E 2}, a_{E}, p_{E}, a_{A}, p_{T}, T_{1}(0), T_{1}^{*}(0)$, $\left.T_{2}(0)\right\}$. We found that the model accurately fit the data for Patient 1 when using several different values of $\gamma$ between 0 and 2, and in particular for $\gamma=1.2$ (see Figure 2). Below, we argue the need to set $\gamma=1.2$ in order to accurately compute standard errors for the parameter estimates.

\section{B. Analysis of the statistical error model}

A key assumption in our inverse problem methodology is that the statistical error model is correct. In particular, the errors $e_{j}^{i}$ in equations (10) and (11) are taken as i.i.d. for $j=1,2$. Incorrect assumptions on these errors can render downstream uncertainty quantification invalid, despite the ability of the mathematical model to fit the data accurately. We analyzed the residual plots that resulted from conducting an inverse problem to test the validity of our statistical error model for a range of $\gamma$ values between 0 and 2. Fanin or fan-out patterns with respect to time or the model solution typically indicate non-i.i.d. residuals. We found that the residual patterns for the viral load varied with $\gamma$, whereas the residuals for the CD4 $\mathrm{T}$ cells were consistently random. Interestingly, both a constant error model $(\gamma=0)$ and a relative error model $(\gamma=1)$ resulted in fan-out patterns with respect to the model solution, indicating the need to increase $\gamma$ further. We performed inverse problems for several values of $\gamma$ between 1 and 2 and found that with $\gamma=1.2$ the residuals showed no pattern with respect to the model solution (Figure 3), whereas higher values of $\gamma$ displayed fan-in patterns with respect to the model solution. Thus, we subsequently set $\gamma=1.2$ to perform the parameter uncertainty quantification.
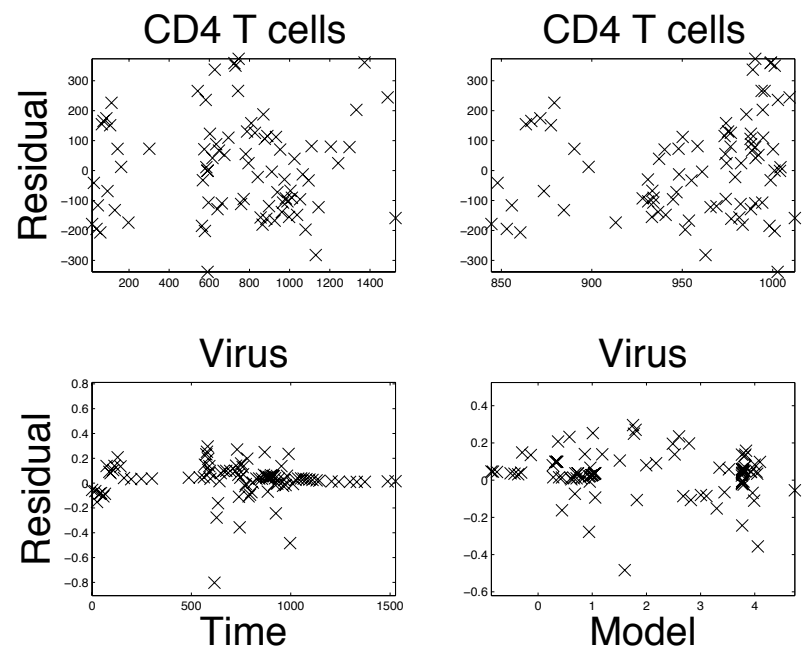

Fig. 3. Residual plots using $\gamma=1.2$.

\section{Parameter uncertainty quantification}

We performed uncertainty quantification using bootstrapping on the parameters we estimated for Patient 1 from 
Banks et al [1]. As discussed above, both the original parameter estimates and the bootstrapping estimates were derived using $\gamma=1.2$ for our statistical error model. Each of the resulting 15 bootstrap distributions and corresponding confidence intervals were narrow (see Figure 4, Table I). The most variable parameter was $T_{2}(0)$, which had a standard error of $<7 \%$ the size of its estimate. These results indicate that the timing and cardinality of the longitudinal data set for Patient 1 are sufficient to generate a reasonable confidence in parameter estimates.
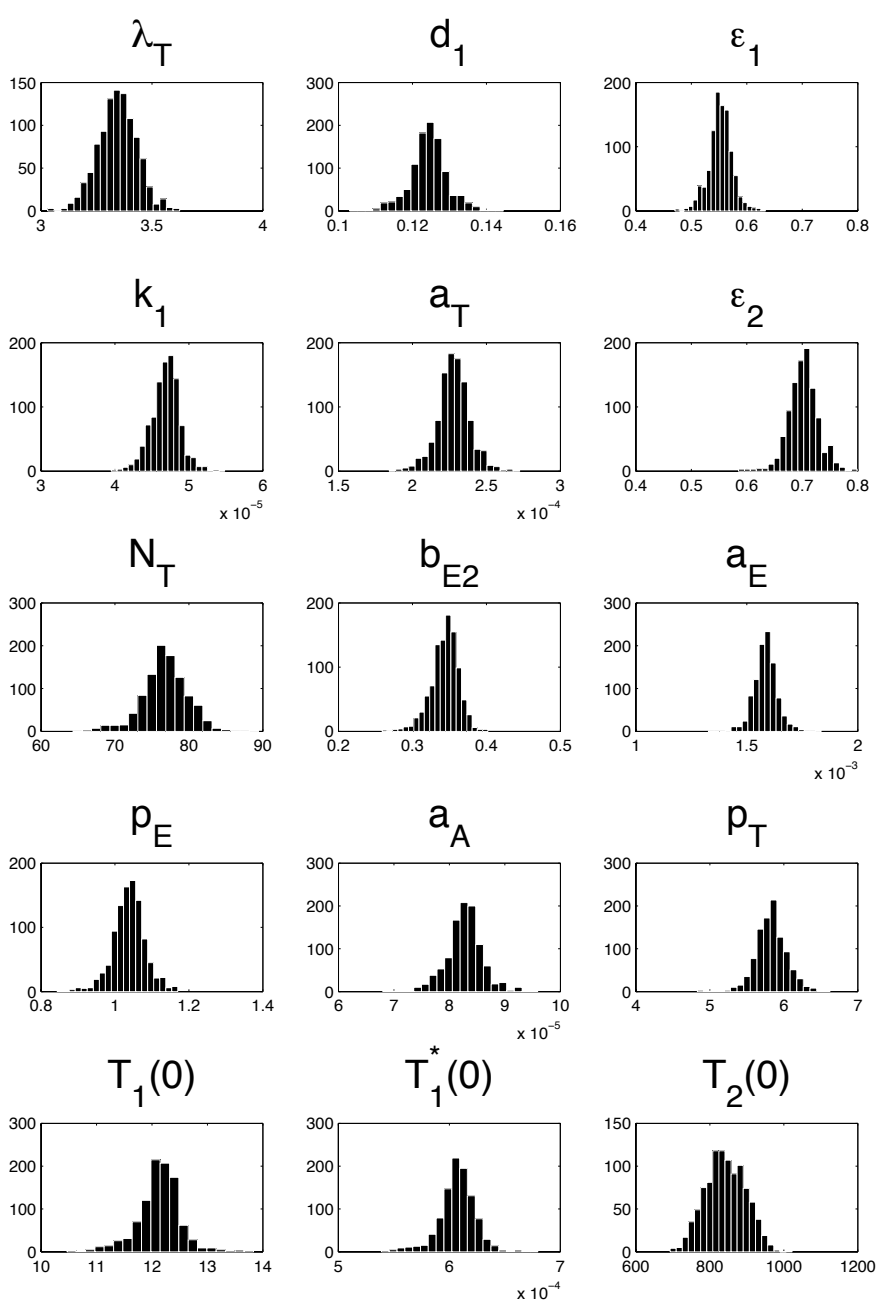

Fig. 4. Parameter distributions from bootstrapping for the patient 1 parameter estimates.

\section{Correlations between parameter estimates}

We used the set of 1000 parameter estimates from bootstrapping to test for correlations between each of the 15 parameters we estimated. To test for correlation between parameters, we computed $R^{2}$ using ordinary least-squares linear regression.

\begin{tabular}{|c|c|c|c|}
\hline Parameter & Estimate & SE $_{\text {Boot }}$ & CI95 Boot \\
\hline$\lambda_{T}$ & 3.2828 & 0.088098 & $(3.1721,3.5175)$ \\
$d_{1}$ & 0.12315 & 0.0050991 & $(0.1144,0.13439)$ \\
$\varepsilon_{1}$ & 0.54379 & 0.020952 & $(0.51172,0.59385)$ \\
$k_{1}$ & $4.77 \mathrm{E}-05$ & $1.91 \mathrm{E}-06$ & $(4.31 \mathrm{E}-05,5.06 \mathrm{E}-05)$ \\
$a_{T}$ & 0.00021986 & $1.11 \mathrm{E}-05$ & $(2.062 \mathrm{E}-05,2.496 \mathrm{E}-05)$ \\
$\varepsilon_{2}$ & 0.69237 & 0.027206 & $(0.64843,0.75508)$ \\
$N_{T}$ & 75.306 & 3.103 & $(70.686,82.85)$ \\
$b_{E 2}$ & 0.35723 & 0.018793 & $(0.30686,0.38053)$ \\
$a_{E}$ & 0.0015925 & $5.33 \mathrm{E}-05$ & $(1.480 \mathrm{E}-03,1.689 \mathrm{E}-03)$ \\
$p_{E}$ & 1.0559 & 0.043749 & $(0.95222,1.1237)$ \\
$a_{A}$ & $8.25 \mathrm{E}-05$ & $3.24 \mathrm{E}-06$ & $(7.64 \mathrm{E}-05,8.91 \mathrm{E}-05)$ \\
$p_{T}$ & 5.6844 & 0.20974 & $(5.4198,6.242)$ \\
$T_{1}(0)$ & 11.912 & 0.40333 & $(11.344,12.925)$ \\
$T_{1}^{*}(0)$ & 0.00059364 & $1.68 \mathrm{E}-05$ & $(5.743 \mathrm{E}-04,6.40 \mathrm{E}-04)$ \\
$T_{2}(0)$ & 828.93 & 53.739 & $(736.65,947.31)$ \\
\hline
\end{tabular}

TABLE I

STANDARD ERRORS AND 95\% CONFIDENCE INTERVALS FROM BOOTSTRAPPING FOR THE PATIENT 1 PARAMETER ESTIMATES. THESE VALUES CORRESPOND TO THE DISTRIBUTIONS IN FIGURE 4.

Overall, the levels of correlation between any 2 parameters were not significant (Figure 5). The largest four correlations were between $\left\{\varepsilon_{2}, b_{E 2}\right\}\left(R^{2}=.219\right),\left\{\varepsilon_{2}, a_{E}\right\}\left(R^{2}=\right.$ $.197),\left\{\varepsilon_{2}, p_{E}\right\}\left(R^{2}=.1726\right)$, and $\left\{a_{T}, b_{E 2}\right\}\left(R^{2}=.164\right)$. Although these correlations are not considered highly significant, they do reflect non-intuitive interactions between drug efficacy, immune effector and memory compartments, and the latent reservoir. For example, the slight correlation between $a_{T}$ and $b_{E 2}$ may suggest a connection between the homeostatic control of the memory CD8 $\mathrm{T}$ cells and the reactivation of latently infected $\mathrm{CD} 4 \mathrm{~T}$ cells from the resting state.

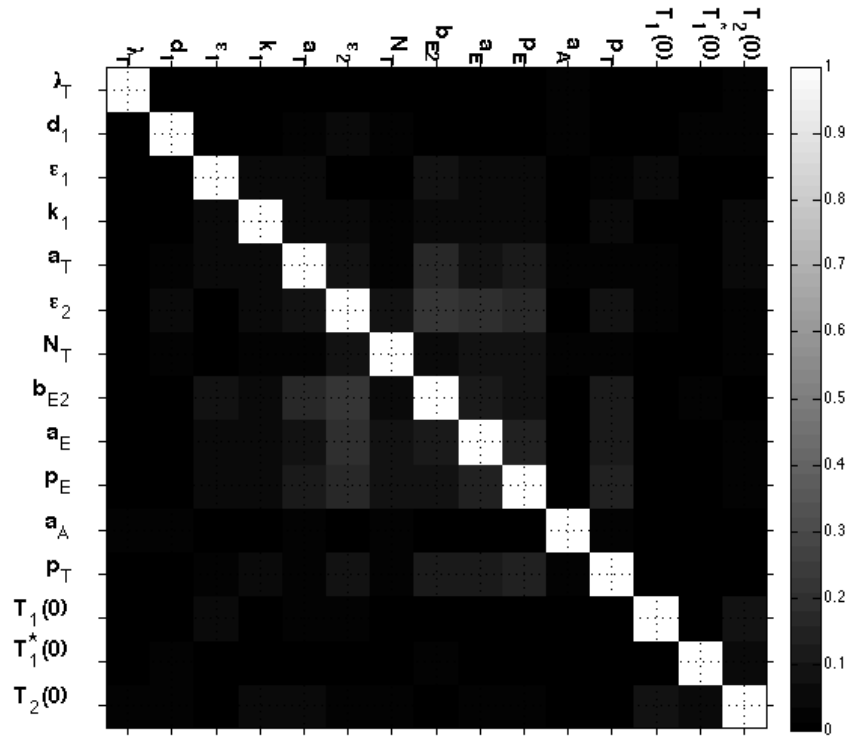

Fig. 5. Correlation between 15 parameters from 1000 bootstrapping estimates. The color indicates the value of $R^{2}$ between two parameters.

\section{Discussion}

In addition to being accurate with respect to the underlying biology and clinical data, we showed that the HIV-1 model by Banks et al in [1] can be used to estimate patient-specific 
parameters with a high level of certainty. Confidence in these parameters is essential in using a mathematical model to interpret clinical data, since they describe the kinetics of the infection, drug efficacy, and proliferative capacity of the immune system. Other patients within the study of STIs in [1] differ in several respects from the patient that we analyzed in this work. It will be informative to test whether the results from our uncertainty analysis here extend to patients with more or less ART interruptions, different mean CD4 T cell counts, and different viral set points when taken off ART.

There has been recent interest in quantitating the contribution of latent reservoirs to the re-emergence of HIV-1 infection after ART cessation [7], [23]. In particular, there are some patients for which ART has successfully suppressed the HIV-1 antigen for $>10$ years (Eric Rosenberg, personal communication). However, the HIV-1 virus has not been eradicated in these patients, as they still have detectable levels of HIV-1 production in CD4 T cells that are thought to be reservoirs for latent HIV-1. If the modeling effort described here has similar results for these patients, we could estimate parameters that describe the capacity of latent cells to reseed the viral infection. If parameter uncertainty is higher for these patients, we could still determine which parameters are unidentifiable, which would inform how the Banks et al model or clinical measurements might be refined.

Our analysis of the correlation between estimated parameters indicate that all of the differences in these parameters between patients reflect true distinctions in their immune system and HIV-1 replication kinetics. If correlations did exist, then some parameters could be different between patients only because they are correlated with parameters that are truly different. Correlations can also indicate the potential for difficulties in applying asymptotic theory to uncertainty quantification. Computing asymptotic standard errors for this model and data set would be very useful, since asymptotic standard errors can be computed much more quickly than by bootstrapping [14] (which took 350 computing hours for Patient 1). The lack of correlations between parameters in our results indicate that asymptotic theory might readily be applied to this model. In future work, we will compute asymptotic standard errors and compare them with the bootstrapping standard errors reported here.

\section{ACKNOWLEDGMENTS}

The authors are grateful to Shuhua $\mathrm{Hu}$ and Eric Rosenberg for helpful conversations.

\section{REFERENCES}

[1] H. T. Banks, M. Davidian, S. Hu, G. M. Kepler, and E. S. Rosenberg, "Modelling HIV immune response and validation with clinical data," Journal of biological dynamics, vol. 2, no. 4, pp. 357-385, Oct. 2008.

[2] S. Lodi, L. Meyer, A. D. Kelleher, M. Rosinska, J. Ghosn, M. Sannes, and K. Porter, "Immunovirologic control 24 months after interruption of antiretroviral therapy initiated close to HIV seroconversion," Archives of internal medicine, vol. 172, no. 16, pp. 1252-1255, Sept. 2012.

[3] B. Max and R. Sherer, "Management of the adverse effects of antiretroviral therapy and medication adherence," Clinical infectious diseases: an official publication of the Infectious Diseases Society of America, vol. 30 Suppl 2, pp. S96-116, June 2000.
[4] S. H. Abdella, N. T. Wabe, and E. A. Yesuf, "Management of common adverse effects in the era of highly active antiretroviral therapy in south east ethiopia," North American journal of medical sciences, vol. 3, no. 11, pp. 499-502, Nov. 2011.

[5] M. Egger, M. May, G. Chłne, A. N. Phillips, B. Ledergerber, F. Dabis, D. Costagliola, A. D'Arminio Monforte, F. de Wolf, P. Reiss, J. D. Lundgren, A. C. Justice, S. Staszewski, C. Leport, R. S. Hogg, C. A Sabin, M. J. Gill, B. Salzberger, J. A. C. Sterne, and ART Cohort Collaboration, "Prognosis of HIV-1-infected patients starting highly active antiretroviral therapy: a collaborative analysis of prospective studies," Lancet, vol. 360, no. 9327, pp. 119-129, July 2002.

[6] J. N. Blankson, D. Persaud, and R. F. Siliciano, "The challenge of viral reservoirs in HIV-1 infection," Annual review of medicine, vol. 53, pp. 557-593, 2002.

[7] A. Alexaki, Y. Liu, and B. Wigdahl, "Cellular reservoirs of HIV-1 and their role in viral persistence," Current HIV research, vol. 6, no. 5, pp. 388-400, Sept. 2008.

[8] M. C. Strain, S. J. Little, E. S. Daar, D. V. Havlir, H. F. Gunthard, R. Y. Lam, O. A. Daly, J. Nguyen, C. C. Ignacio, C. A. Spina, D. D. Richman, and J. K. Wong, "Effect of treatment, during primary infection, on establishment and clearance of cellular reservoirs of HIV1," The Journal of infectious diseases, vol. 191, no. 9, pp. 1410-1418, May 2005.

[9] N. Ngo-Giang-Huong, C. Deveau, I. Da Silva, I. Pellegrin, A. Venet, M. Harzic, M. Sinet, J. F. Delfraissy, L. Meyer, C. Goujard, C. Rouzioux, and Frnech PRIMO Cohort Study Group, "Proviral HIV-1 DNA in subjects followed since primary HIV-1 infection who suppress plasma viral load after one year of highly active antiretroviral therapy," AIDS (London, England), vol. 15, no. 6, pp. 665-673, Apr. 2001.

[10] C. Dalmasso, W. Carpentier, L. Meyer, C. Rouzioux, C. Goujard, M.-L. Chaix, O. Lambotte, V. Avettand-Fenoel, S. Le Clerc, L. D. de Senneville, C. Deveau, F. Boufassa, P. Debr, J.-F. Delfraissy, P. Broet, I. Theodorou, and ANRS Genome Wide Association 01, "Distinct genetic loci control plasma HIV-RNA and cellular HIVDNA levels in HIV-1 infection: the ANRS genome wide association 01 study," PloS one, vol. 3, no. 12, p. e3907, 2008.

[11] B. Descours, V. Avettand-Fenoel, C. Blanc, A. Samri, A. Mlard, V. Supervie, I. Theodorou, G. Carcelain, C. Rouzioux, B. Autran, and ALT ANRS CO15 Study Group, "Immune responses driven by protective human leukocyte antigen alleles from long-term nonprogressors are associated with low HIV reservoir in central memory CD4 t cells," Clinical infectious diseases: an official publication of the Infectious Diseases Society of America, vol. 54, no. 10, pp. 1495-1503, May 2012.

[12] O. Lambotte, F. Boufassa, Y. Madec, A. Nguyen, C. Goujard, L. Meyer, C. Rouzioux, A. Venet, J.-F. Delfraissy, and SEROCOHEMOCO Study Group, "HIV controllers: a homogeneous group of HIV-1-infected patients with spontaneous control of viral replication," Clinical infectious diseases: an official publication of the Infectious Diseases Society of America, vol. 41, no. 7, pp. 1053-1056, Oct. 2005.

[13] J. Ananworanich, C. Vandergeeten, N. Chomchey, and N. Chomont, "Early ART intervention restricts the seeding of the HIV reservoir in long-lived central memory CD4 $\mathrm{t}$ cells," Program and abstracts of the 20 Conference on Retroviruses and Opportunistic Infections, Atlanta, $G A$, p. abstract no. 47, 2013.

[14] H. T. Banks, K. Holm, and D. Robbins, "Standard error computations for uncertainty quantification in inverse problems: Asymptotic theory vs. bootstrapping," Mathematical and computer modelling, vol. 52, no. 9-10, pp. 1610-1625, Nov. 2010.

[15] M. Catalfamo, C. Wilhelm, L. Tcheung, M. Proschan, T. Friesen, J.-H. Park, J. Adelsberger, M. Baseler, F. Maldarelli, R. Davey, G. Roby, C. Rehm, and C. Lane, "CD4 and CD8 t cell immune activation during chronic HIV infection: roles of homeostasis, HIV, type i IFN, and IL7," Journal of immunology (Baltimore, Md.: 1950), vol. 186, no. 4, pp. 2106-2116, Feb. 2011.

[16] R. M. Welsh, K. Bahl, H. D. Marshall, and S. L. Urban, "Type 1 interferons and antiviral CD8 t-cell responses," PLoS pathogens, vol. 8, no. 1, p. e1002352, Jan. 2012.

[17] T. H. Mogensen, J. Melchjorsen, C. S. Larsen, and S. R. Paludan, "Innate immune recognition and activation during HIV infection," Retrovirology, vol. 7, p. 54, 2010.

[18] N. Zhang and M. J. Bevan, "CD8(+) t cells: foot soldiers of the immune system," Immunity, vol. 35, no. 2, pp. 161-168, Aug. 2011.

[19] L. Sompayrac, How the Immune System Works. Wiley, Jan. 2003. 
[20] L. Shan, K. Deng, N. S. Shroff, C. M. Durand, S. A. Rabi, H.-C. Yang, H. Zhang, J. B. Margolick, J. N. Blankson, and R. F. Siliciano, "Stimulation of HIV-1-specific cytolytic t lymphocytes facilitates elimination of latent viral reservoir after virus reactivation," Immunity, vol. 36, no. 3, pp. 491-501, Mar. 2012.

[21] R. M. van der Sluis, T. van Montfort, G. Pollakis, R. W. Sanders, D. Speijer, B. Berkhout, and R. E. Jeeninga, "Dendritic cell-induced activation of latent HIV-1 provirus in actively proliferating primary $\mathrm{t}$ lymphocytes," PLoS pathogens, vol. 9, no. 3, p. e1003259, Mar. 2013.

[22] H. T. Banks and H. T. Tran, Mathematical and Experimental Modeling of Physical and Biological Processes. CRC Press, Mar. 2011.

[23] R. F. Siliciano and W. C. Greene, "HIV latency," Cold Spring Harbor Perspectives in Medicine:, vol. 1, no. 1, Sept. 2011. 